\title{
Exposition professionnelle dans les usines de retraitement *
}

\author{
P. HENRY ** \\ (Manuscrit reçu le 7 février 1986)
}

\begin{abstract}
RÉSUME
L'exposition professionnelle dans les usines de retraitement françaises de La Hague et de Marcoule ne peut être abordée sans rappeler les risques potentiels associés aux activités de retraitement caractérisées par une grande diversité et une évolutivité liées au combustible et à la nature des opérations mécaniques et chimiques qui se déroulent au cours des diverses phases du procédé, ou lors des diverses activités situées en amont ou en aval du procédé. Les résultats concernant l'exposition professionnelle dans les deux usines et son évolution au cours des douze dernières années sont examinés : doses moyennes annuelles des agents travaillant dans quatre secteurs sensibles et exposition de l'ensemble des travailleurs. L'évolution, dans l'ensemble favorable, de ces résultats est brièvement analysée. En conclusion, sont évoqués les principes directeurs qui se sont dégagés de l'expérience et qui permettront de réduire encore, dans les futures usines. l'exposition professionnelle due au retraitement.
\end{abstract}

\section{ABSTRACT}

Occupational exposure in the french fuel reprocessing plants of La Hague and Marcoule cannot be studied without recalling the potential risks associated with reprocessing activities characterised by a large variety and evolutivity due to both the fuel and the nature of the mechanical and chemical processes or other up- or downstream activities. The results of occupational exposure in both plants and its evolution during the last twelve years are examined : annual mean doses for workers employed in four sensitive sectors and exposure of all the workers. The evolution of the results, favourable as a whole, is analysed. As a conclusion, the guiding principles gained from experience, which will allow to further reduce occupational exposure from reprocessing, are stated.

* Communication présentée au Congrès franco-italien "Les expositions professionnelles - aspects méthodologiques et expériences pratiques" organisé à Nice du 29 au 31 janvier 1986, par la Société française de radioprotection et l'Associazione italiana di protezione contro le radiazioni.

** COGEMA - Branche Retraitement, BP 4, 78141 Velizy-Villacoublay Cedex. 


\section{INTRODUCTION}

L'exposition professionnelle dans les usines de retraitement et son évolution dans le temps pour les usines françaises de La Hague et de Marcoule ne peuvent être abordées sans rappeler les caractéristiques des opérations de retraitement et les risques potentiels qui y sont associés. Ce rappel constituera la première partie de cet exposé. La deuxième partie traitera des résultats concernant l'exposition professionnelle et son évolution au cours des douze dernières années.

\section{LE RETRAITEMENT : CARACTERISTIQUES ET RISQUES POTENTIELS}

Le but du retraitement des combustibles irradiés, schématisé dans la figure 1, est de séparer les produits nobles à recycler -plutonium et uranium- des déchets non récupérables constitués essentiellement par les matériaux de structure et les produits de fission. Les opérations nécessaires pour atteindre ce but présentent, sur le plan des risques d'exposition, les caractéristiques essentielles suivantes:

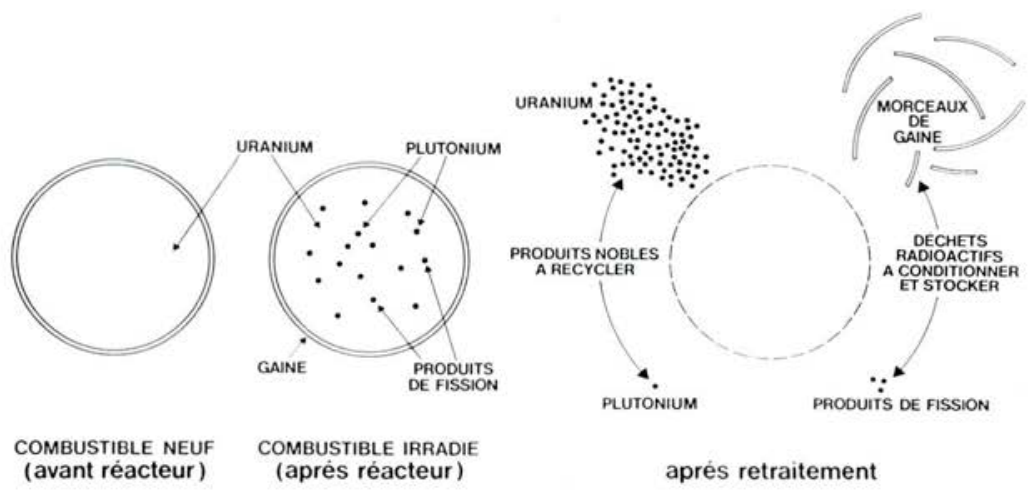

Fig.1. - Le but du retraitement.

\section{I.1. Diversité des combustibles retraités et de l'activité des radionu- cléides contenus}

On peut regrouper les différentes sortes de combustibles irradiés retraités en France en trois catégories:

1. les combustibles "plutonigènes" qui proviennent de réacteurs fournissant le plutonium destiné à l'armement nucléaire :

2. les combustibles "électrogènes" qui proviennent des différents types de centrales nucléaires en fonctionnement :

- réacteurs à uranium naturel graphite gaz (UNGG). 
- réacteurs à eau lourde (EL),

- réacteurs à eau légère ordinaire (EOr) comprenant : les réacteurs à eau pressurisée (REP ou PWR), les réacteurs à eau bouillante (REB ou BWR) ;

- réacteurs surgénérateurs à neutrons rapides (NR ou FBR) ;

3. des combustibles divers provenant notamment des réacteurs de recherche, cités simplement pour mémoire en raison de la quantité limitée des combustibles retraités.

Les caractéristiques, très variées, des principaux combustibles électrogènes sont indiquées dans le tableau I.

Les radionucléides présents dans les combustibles, et qui sont la source des risques d'exposition, sont constitués d'émetteurs $\beta, \gamma$ (produits de fission, PF) et d'émetteurs $\alpha$ (essentiellement plutonium et transplutoniens). Il faut y ajouter les produits d'activation contenus dans les matériaux de structure et les gaines. La teneur en radionucléides est très dépendante des caractéristiques des combustibles irradiés dont les principales sont le taux de combustion $\left(\mathrm{MWJ} . \mathrm{t}^{-1}\right.$ ) et le temps de refroidissement depuis le déchargement du réacteur.

TABLEAU I

Combustibles électrogènes

\begin{tabular}{|c|c|c|c|}
\hline & GG & EOr & NR \\
\hline $\begin{array}{l}\text { Combustible } \\
\text { Matière } \\
\text { Nature chimique } \\
\% \text { fissile }\end{array}$ & $\begin{array}{c}\text { U } \\
\text { Métal } \\
0,7\end{array}$ & $\begin{array}{c}\mathrm{U} \\
\text { Oxyde } \\
3\end{array}$ & $\begin{array}{c}\text { U Pu } \\
\text { Oxyde } \\
>10\end{array}$ \\
\hline $\begin{array}{l}\text { Elément } \\
\text { Poids }(\mathrm{kg}) \\
\text { Longueur }(\mathrm{m}) \\
\text { Gainage }\end{array}$ & $\begin{array}{c}10 \\
0.60 \\
\text { Magnésium }\end{array}$ & $\begin{array}{c}150 \text { à } 520 \\
4 \text { à } 5 \\
\text { Zircalloy }\end{array}$ & $\begin{array}{c}45 \text { à } 100 \\
3 \\
\text { Acier inox }\end{array}$ \\
\hline $\begin{array}{l}\text { Taux d'irradiation } \\
(\mathrm{MWJ} / \mathrm{t})\end{array}$ & 5000 & 33000 & 100000 \\
\hline
\end{tabular}

Le tableau II donne la composition très simplifiée de trois combustibles types. Lorsque le taux de combustion croît, on observe une augmentation :

- de l'activité globale $\beta, \gamma$ due aux PF. Cette augmentation est plus importante pour les émetteurs à vie longue, tels ${ }^{90} \mathrm{Sr}$ et ${ }^{137} \mathrm{Cs}$ en raison de la différence entre les temps de refroidissement des combustibles UNGG (1 an) et des combustibles EOr (3 ans) ;

- de la quantité de plutonium et de l'activité $\alpha$ associée qui croît plus rapidement que la masse. Cette augmentation de l'activité spécifique $\alpha$ du $\mathrm{Pu}$ provient de la modification de sa composition isotopique et de l'influence prépondérante de l'activité du plutonium 238. 
TABLEAU ॥

Composition simplifiée de quelques combustibles types

\begin{tabular}{|c|c|c|c|}
\hline Filière & \multicolumn{2}{|c|}{ UNGG } & \multirow{2}{*}{$\begin{array}{c}\text { EOr } \\
33000\end{array}$} \\
\hline $\begin{array}{l}\text { Taux de combustion } \\
\qquad(\mathrm{MWJ} / \mathrm{t})\end{array}$ & 200 & 4500 & \\
\hline $\begin{array}{l}\text { Temps } \\
\text { de refroidissement } \\
\text { (an) }\end{array}$ & 1 & 1 & 3 \\
\hline $\begin{array}{l}\text { Produits de fission } \\
\qquad(\mathrm{Ci}, \beta, \gamma / \mathrm{T})\end{array}$ & $3,1 \quad 10^{4}$ & $3.710^{5}$ & $7,610^{5}$ \\
\hline dont $\quad{ }^{90} \mathrm{Sr}$ & $\begin{array}{ll}6,4 & 10^{2} \\
6,2 & 10^{2}\end{array}$ & $\begin{array}{ll}1,2 & 10^{4} \\
1,6 & 10^{4}\end{array}$ & $\begin{array}{ll}6,8 & 10^{4} \\
9,8 & 10^{4}\end{array}$ \\
\hline $\begin{array}{l}\text { Plutonium }(\mathrm{g} / \mathrm{t}) \\
\qquad(\mathrm{Ci}, \alpha / \mathrm{t}) \\
\mathrm{Ci}, \alpha / \mathrm{g} \text { de } \mathrm{Pu}\end{array}$ & $\begin{array}{c}1,810^{2} \\
1,210^{1} \\
0,066\end{array}$ & $\begin{array}{c}2,910^{3} \\
3,510^{2} \\
0,12\end{array}$ & $\begin{array}{cc}9,7 & 10^{3} \\
3,9 & 10^{3} \\
0,4 & \end{array}$ \\
\hline
\end{tabular}

\section{I.2. Opérations multiples et de nature complexe sur des matières radio- actives aisément dispersables (gaz, liquides, poudres)}

La figure 2 présente un schéma simplifié des opérations de retraitement qui comprennent :

- des opérations "amont" :

transport des combustibles irradiés des réacteurs vers l'usine de reretraitement,

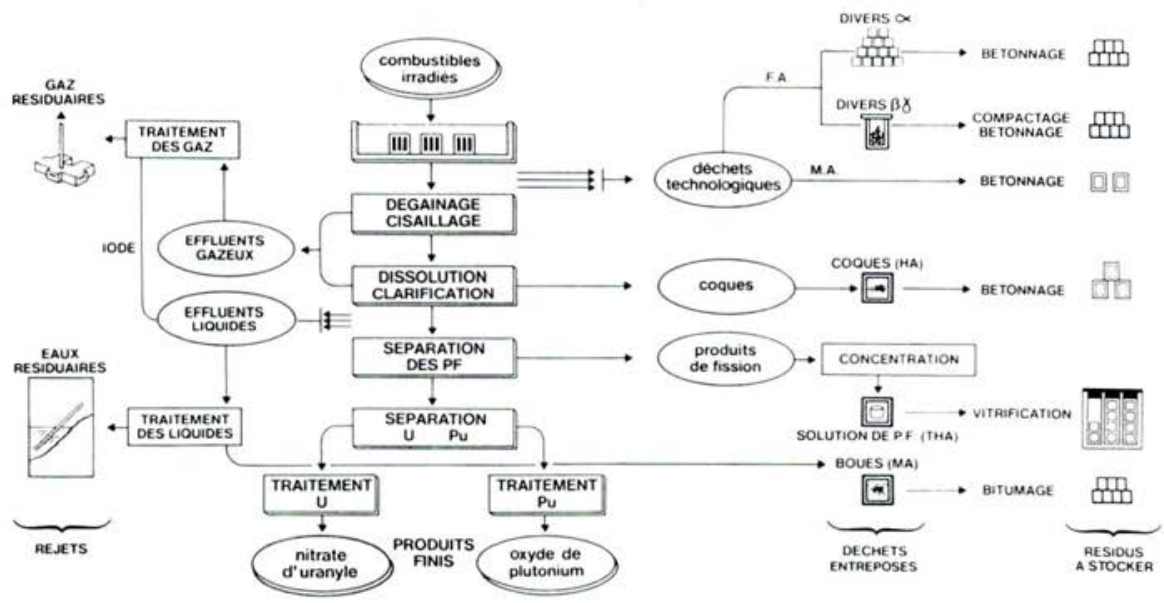

Fig. 2. - Schéma des opérations de retraitement. 
déchargement et stockage en piscine des éléments en attente de retraitement ;

- le procédé de retraitement :

opérations mécaniques de démantèlement des assemblages, opérations mécaniques ou chimiques de mise à nu des combustibles ;

opérations chimiques (procédé Purex) de dissolution, d'extraction par solvant, de purification et de transformation, suivies du conditionnement des matières recyclables: uranium et plutonium ;

sont également rattachés au procédé le traitement d'épuration des effluents gazeux et le stockage des solutions concentrées de produits de fission ;

- des opérations "aval" :

. traitement d'épuration des effluents liquides,

entreposage et conditionnement des déchets solides,

transport des produits finis vers les utilisateurs et des déchets conditionnés vers les centres de stockage:

- des opérations diverses d'entretien, de décontamination, d'intervention ainsi que les analyses nécessaires au suivi du procédé.

Ces activités nécessitent des ateliers nombreux, de caractéristiques et de tailles très variées, ainsi que des équipes spécialisées.

L'expérience acquise dans les usines montre que les points les plus sensibles vis-à-vis des risques d'exposition sont :

- les opérations mécaniques en tête du procédé (démantèlement, dégainage) ;

- les opérations par voie sèche sur le plutonium en fin de procédé (atelier moyenne activité plutonium) ;

- le traitement des effluents liquides;

- les travaux et interventions effectués dans le cadre de la maintenance normale des installations ou à la suite d'incidents de fonctionnement d'origine matérielle ou humaine.

\section{I.3. Caractère évolutif des activités et de la production}

Le tableau III indique les grandes lignes de l'évolution des activités de retraitement dans les usines de Marcoule (UP1) et de La Hague (UP2), créées par le Commissariat à l'énergie atomique (CEA), mais qui, depuis 1976 appartiennent à la Compagnie générale des matières nucléaires (Cogéma), filiale du CEA.

UP1 a démarré en 1958 pour le retraitement des combustibles plutonigènes et UP2 en 1966 pour le retraitement des combustibles de la filière UNGG. A la suite de l'abandon, vers la fin des années 60 , de la filière UNGG au profit de la filière à eau légère, l'usine UP2 a dû s'adapter au retraitement de ce type de combustible en créant un nouvel atelier HAO (haute activité oxydes) en tête du procédé. Depuis 1976, UP2 retraite les combustibles oxydes en quantité croisante, le retraitement des combustibles UNGG, qui se terminera en fin de siècle, étant progressivement et bientôt en totalité transféré à l'usine UP1. 
TABLEAU III

Evolution des activités de retraitement à Marcoule (UP1) et à La Hague (UP2)

\begin{tabular}{|c|c|c|c|c|c|c|}
\hline & ilière & Réacteur & $\begin{array}{c}\text { Taux } \\
\text { d'irradiation } \\
\text { maximal } \\
(\mathrm{MWJ} / \mathrm{t})\end{array}$ & $\begin{array}{c}\text { Année } \\
\text { de mise } \\
\text { en } \\
\text { retraitement }\end{array}$ & Usine & $\begin{array}{c}\text { Capacité } \\
\text { en } \\
\text { t. } \text { an }^{-1}\end{array}$ \\
\hline plut & $\begin{array}{l}\text { G } \\
\text { pnigène }\end{array}$ & $\begin{array}{c}\text { G } \\
\text { Marcoule }\end{array}$ & 1000 & 1958 & UP1 & \\
\hline \multirow{3}{*}{ 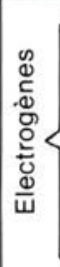 } & UNGG & $\begin{array}{c}\text { EdF } \\
\text { Vandellos }\end{array}$ & 6500 & $\begin{array}{l}1966 \\
1976\end{array}$ & $\begin{array}{l}\text { UP2 } \\
\text { UP1 }\end{array}$ & $\begin{array}{l}800 \\
800\end{array}$ \\
\hline & EOr & $\begin{array}{c}\text { REP \& REB } \\
\text { EdF } \\
+ \text { étranger }\end{array}$ & 33000 & 1976 & UP2 & 400 \\
\hline & NR & Phénix & 70000 & 1979 & UP2 & 10 \\
\hline
\end{tabular}

L'usine actuelle UP2 400 étant insuffisante pour les besoins futurs, la Cogéma a entrepris la construction à La Hague de deux nouvelles usines de retraitement de combustibles eau légère, UP2 800 et UP3 d'une capacité totale de 1600 tonnes.an'1 et d'une nouvelle station de traitement des effluents (STE 3).

L'afflux des combustibles UNGG a également obligé l'usine UP1 à s'adapter en construisant, notamment, une nouvelle installation de dégainage (MAR 400) qui a démarré en 1983, et un nouvel atelier moyenne activité en fin de procédé. Une nouvelle station de traitement des effluents est également prévue ; il faut citer, en outre, le démarrage en 1978 d'un atelier de vitrification des produits de fission.

Parmi les activités annexes du retraitement dans les deux usines, il faut mentionner:

- Pour UP1, le retraitement de combustibles MTR (réacteurs de recherche), de combustibles de la filière à eau lourde (EL4 arrêté en 1985) ainsi que des couvertures fertiles du réacteur prototype surgénérateur Phénix ;

- pour UP2, le retraitement de 1979 à 1984 d'une partie du cœur de Phénix, l'autre partie étant retraitée dans l'atelier TOP du CEA implanté à Marcoule.

Les figures 3 et 4 montrent l'évolution annuelle du tonnage de combustibles UNGG (à l'exception des plutonigènes) et eau légère retraités dans les deux usines. La figure 5 indique les résultats de la production exprimée en énergie d'irradiation (MWJ et MWe.an) des combustibles retraités à La Hague. Ces figures illustrent la croissance importante des tonnages et des MWJ retraités chaque année, qui se traduit par une augmentation parallèle des risques potentiels. 


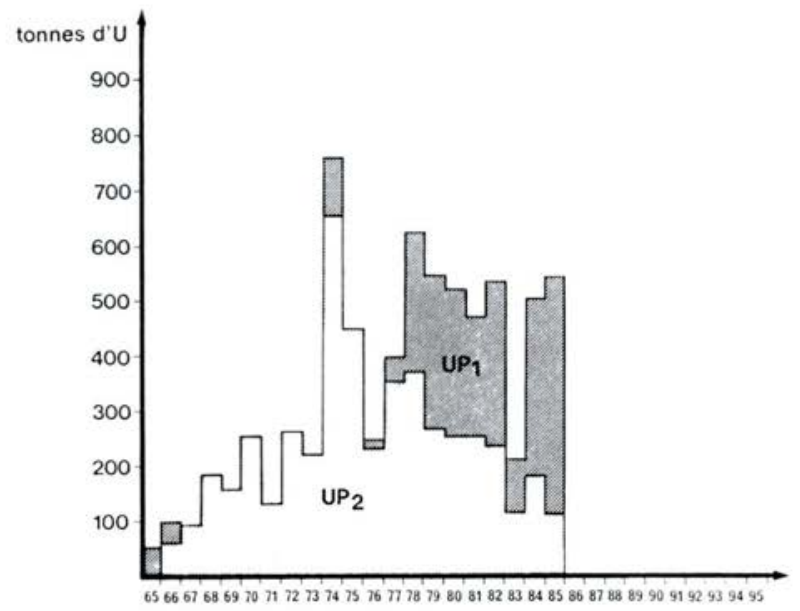

Fig. 3. Retraitement des combustibles UNGG (EdF + Hifrensa).

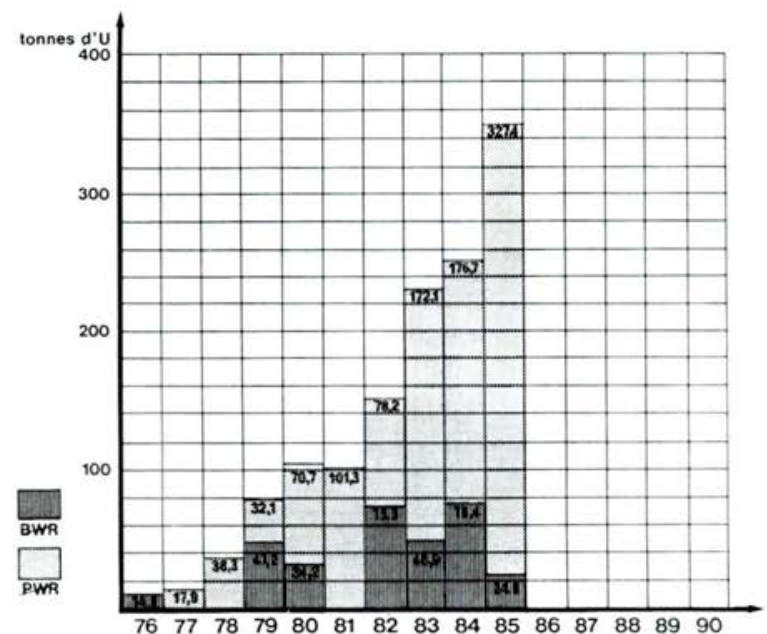

Fig. 4. - Retraitement annuel des combustibles "eau ordinaire", au 31.12.85. 


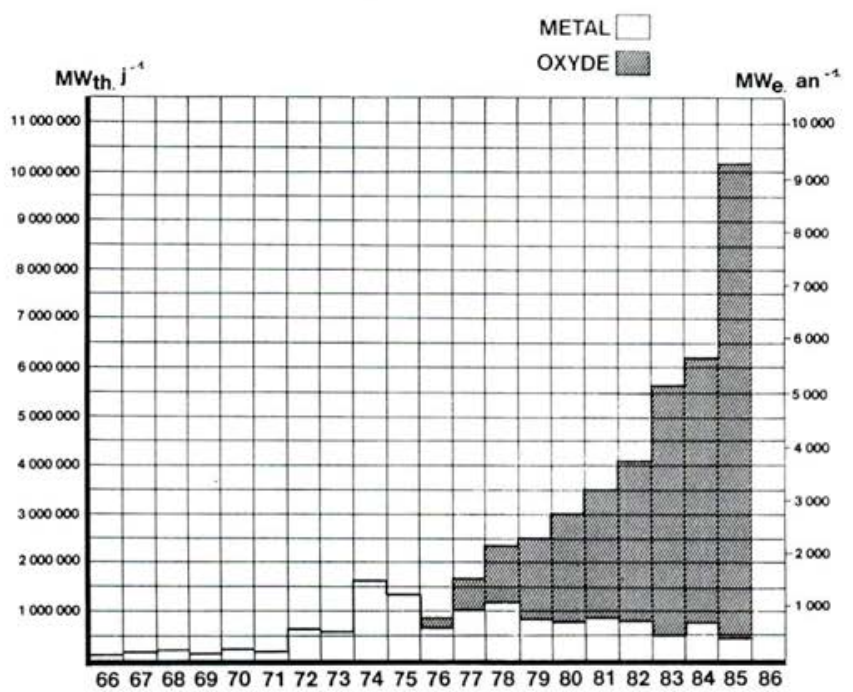

Fig. 5 - Energie d'irradiation exprimée en $M W_{t h .} .^{-1}$ et en $M W_{e}{ }^{a n^{-1}}$.

\section{EXPOSITION PROFESSIONNELLE DANS LE RETRAITEMENT}

Les risques radiologiques encourus par le personnel concernent l'exposition externe (irradiation) et l'exposition interne (contamination). La manipulation de matières fissiles induit un risque d'accident de criticité qui serait susceptible d'entraîner une surexposition importante, mais les précautions prises en ce domaine sont telles quaucun accident de ce type n'est à déplorer depuis le démarrage des deux usines. De même, l'efficacité des barrières de confinement, la précocité de la détection et de l'alarme en cas d'incident, l'utilisation systématique de moyens de protection individuels en cas d'intervention font que les cas de contamination interne sont rares et peu importants. Leur contribution à l'exposition du personnel peut être considérée comme négligeable.

$\mathrm{Ne}$ seront donc exposées ici que les données concernant l'exposition externe de l'organisme entier. Seront examinés successivement :

- les doses moyennes annuelles des agents affectés aux quatre secteurs les plus sensibles déjà mentionnés ;

- les différents paramètres caractérisant l'exposition de l'ensemble des travailleurs concernés.

\section{II.1. Exposition des agents travaillant dans les secteurs les plus exposés}

\section{II.1.1. Démantèlement et dégainage des combustibles}

Les opérations portant sur les combustibles UNGG comportent un certain nombre de traitements mécaniques variables suivant les combustibles : déconteneurage, déchemisage, déqueusotage, dégraphitage. La 
gaine magnésium est ensuite séparée du combustible par voie mécanique à Marcoule et par pelage chimique à La Hague. Toutes les opérations mécaniques s'effectuent dans des fosses remplies d'eau, sauf dans le nouvel atelier de Marcoule (MAR 400) qui est entré en fonctionnement en 1983 et où les combustibles sont télémanipulés en cellule. Cependant, par suite des difficultés de démarrage de MAR 400, l'ancien atelier a du être remis en service et les deux installations fonctionnent simultanément ; en conséquence, il est encore trop tôt pour juger le gain dans la réduction des doses que doit apporter le dégainage en cellule.

L'évolution des doses moyennes annuelles du personnel d'exploitation est présentée dans la figure 6. A La Hague comme à Marcoule, mais avec un décalage dans le temps, on a constaté une montée progressive des doses en rapport avec la croissance des MWJ retraités et les difficultés rencontrées : flashes, corrosion des barreaux, ennuis mécaniques, augmentation des débits de doses au niveau du platelage des fosses de travail due à la contamination de l'eau par les poussières mises en suspension. Une nette amélioration s'est produite, due à la maîtrise progressive des problèmes et l'emploi de remèdes appropriés : télémanipulation des combustibles, amélioration du traitement des eaux, mise en place de protections supplémentaires sur le platelage des fosses, augmentation de la fiabilité du matériel.

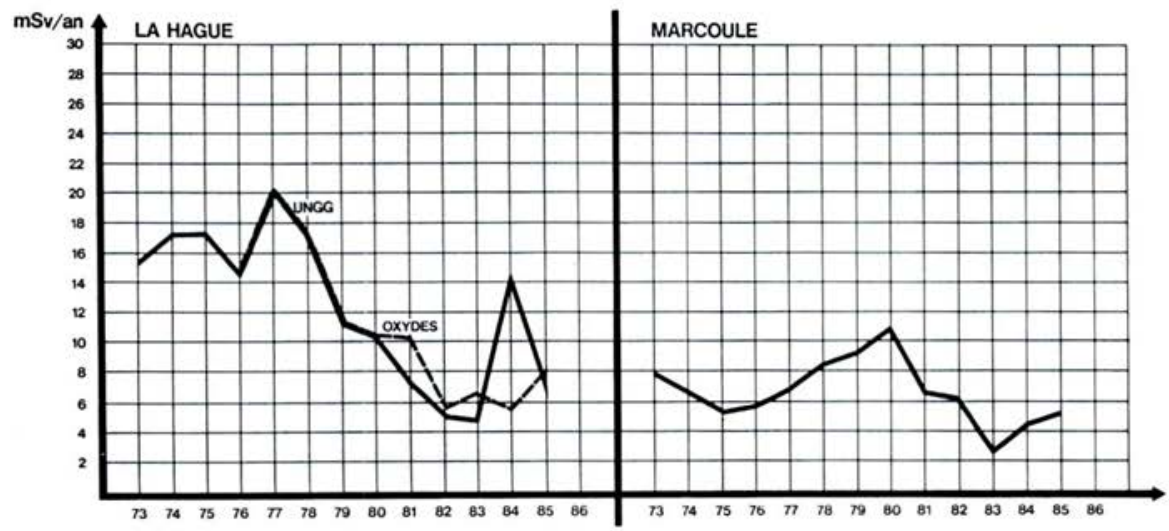

Fig. 6. - Dégainage - Traitements mécaniques : doses moyennes annuelles en mSv.

Sur la courbe de La Hague, la valeur élevée de l'année 1984 est due à une opération "ménage" au cours de laquelle on a effectué la reprise de vieux combustibles UNGG corrodés, ayant fortement contaminé la piscine où ils étaient stockés. La partie de la courbe correspondant aux années 1976 et 1979 représente les doses reçues par le personnel travaillant à la fois au dégainage (UNGG) et au HAO Sud où s'effectue, pour les combustibles à eau légère, le cisaillage des crayons en cellule. A partir de 1980, les doses moyennes des agents travaillant au HAO Sud ont été individualisées. L'essentiel des doses, dans ce secteur, provient des opérations de transfert des coques (morceaux de gaine) très irradiantes, vers le silo de stockage.

La courbe de Marcoule, après le pic de l'année 1980, montre une décroissance très nette des doses, la valeur très basse de 1983 correspondant à une production très réduite. 


\section{II.1.2. Moyenne activité plutonium (MAPu)}

Dans ces ateliers, s'effectuent les opérations de fin de procédé sur le plutonium : conversion du nitrate en oxalate puis en oxyde, mélange et conditionnement ; à Marcoule s'ajoutent, pour les combustibles plutonigènes, des opérations d'élaboration du plutonium métal. Le problème essentiel réside dans la difficulté de conditionnement des poudres à l'intérieur des appareillages du procédé. La dispersion du $\mathrm{PuO}_{2}$ à l'intérieur des boîtes à gants contenant les appareils pose des problèmes complexes de protection dus à la géométrie de la source et aux caractéristiques radioactives du plutonium. A ce stade, le plutonium est, certes, débarrassé de la quasi-totalité des produits de fission, mais il émet lui-même des rayonnements complexes : $\chi, \gamma$ et neutrons provenant de fissions spontanées, de fissions provoquées et de réactions $(\alpha, n)$. Par suite de la modification de la composition isotopique du plutonium, les débits de dose qu'il délivre croissent avec le taux d'irradiation des combustibles dont il est extrait. L'évolution des doses moyennes annuelles est présentée dans la figure 7. En dépit de certaines fluctuations et de l'augmentation des risques potentiels avec la production, on peut observer une stabilisation puis une diminution des doses, très nette au cours des dernières années. Cette diminution correspond à une amélioration des conditions d'exploitation obtenues par la diminution des opérations manuelles (automatisation) et le renforcement des protections contre les neutrons et les rayonnements gamma.

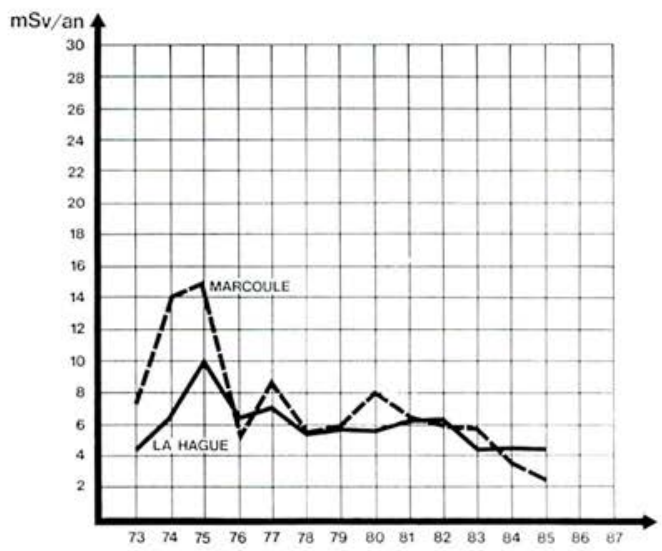

Fig. 7. - Usine de moyenne activité plutonium : doses moyennes annuelles en mSv.

\section{II.1.3. Traitements des effluents liquides}

En dehors des solutions concentrées de produits de fission de très haute activité qui sont stockées avant d'être vitrifiées, le retraitement génère des volumes importants d'effluents liquides de faible ou moyenne activité qui sont dirigés vers la station de traitement des effluents où ils subissent une série de traitements par coprécipitation et floculation. Les eaux résiduaires décantées et filtrées très peu radioactives sont rejetées 
en mer, ou dans le Rhône, suivant des procédures définies dans les arrêtés d'autorisation de rejet. Les boues provenant du traitement ont une destination différente dans les deux établissements :

- à La Hague, elles sont stockées dans des silos prévus à cet effet ;

- à Marcoule, elle font l'objet d'un traitement par enrobage dans du bitume ; les enrobés bitumineux conditionnés dans des fûts métalliques sont ensuite stockés dans des casemates.

L'évolution de l'exposition du personnel d'exploitation de ces installations est présentée dans la figure 8 . Si la courbe de La Hague montre une évolution satisfaisante des doses, celle de Marcoule reflète les grosses difficultés rencontrées lors de l'arrivée des combustibles électrogènes UNGG, beaucoup plus irradiés que ceux provenant des réacteurs plutonigènes. A la vétusté des installations, s'est ajoutée une indisponibilité prolongée de l'atelier d'enrobage des boues. L'accumulation de ces dernières dans des bassins non conçus pour les stocker s'est traduite par une augmentation importante des niveaux d'irradiation. Depuis 1981, on constate une amélioration notable due à la résorption partielle du stock des boues, au renforcement des protections et à certaines modifications concernant des conditions d'exploitation. Cependant, la situation ne pourra être normalisée que lorsque la deuxième chaîne d'enrobage actuellement en construction aura permis la résorption totale des boues. II convient, en outre, de rappeler que les deux établissements disposeront dans un futur proche de nouvelles stations de traitement des effluents.

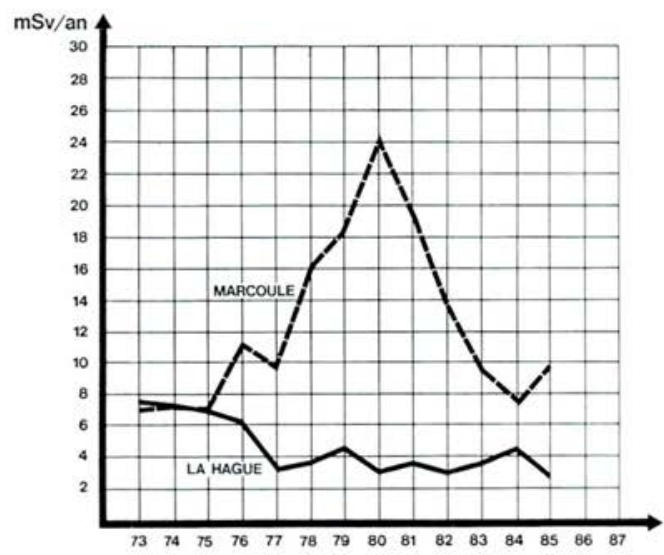

Fig. 8. - Traitement des effluents liquides: doses moyennes annuelles en mSv.

\subsubsection{Décontamination-intervention}

Cette mission est confiée à des équipes mobiles spécialisées dans l'assainissement et qui ont à intervenir dans diverses situations parmi lesquelles on peut citer:

- réparation ou changement de matériels usés ou défectueux ;

- préparation des travaux d'intercampagne concernant l'entretien, les mo- 
difications ou rénovations d'atelier ;

- remise en état des installations après incidents.

L'évolution de l'exposition du personnel dans ce secteur d'activité est montrée dans la figure 9 . On peut constater une réduction progressive des doses qui est le fait des progrès réalisés en ce domaine : amélioration des méthodes de travail (travail à distance), formation poussée et spécialisée du personnel, expérience acquise par les intervenants.

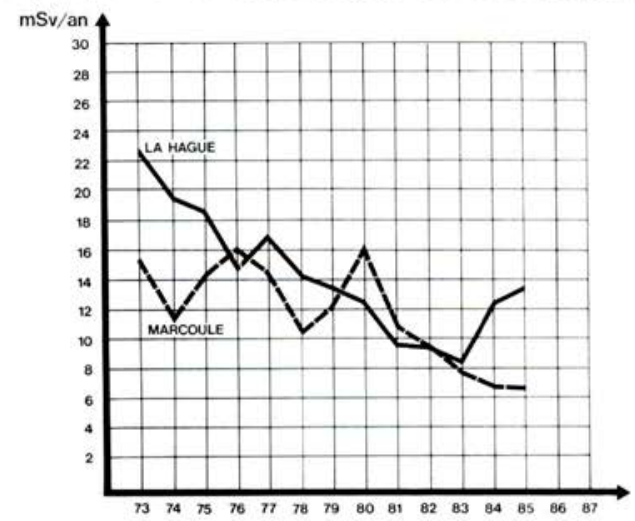

Fig. 9. - Décontamination intervention : doses moyennes annuelles en mSv.

La nette remontée en 1984 et 1985 de la courbe de La Hague est un phénomène conjoncturel en liaison avec l'opération "ménage" sur des combustibles UNGG corrodés et avec d'autres chantiers importants.

\section{II.2. Exposition de l'ensemble des travailleurs}

La figure 10 montre l'évolution des doses collectives totales reçues par l'ensemble du personnel surveillé appartenant à la Cogéma et aux

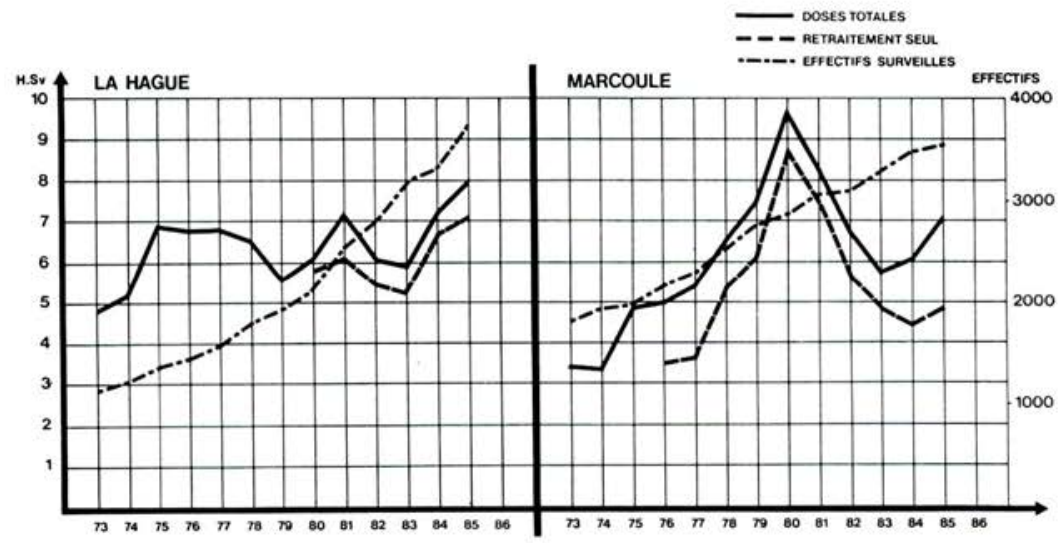

Fig. 10. - Doses collectives totales (Cogéma + entreprises) en H.Sv, et effectifs surveillés. 
entreprises extérieures ainsi que celle des effectifs correspondants. Les courbes inférieures correspondent aux doses dues à l'exploitation et à la maintenance du seul retraitement, déduction faite des doses reçues dans les autres installations, les chantiers de démantèlement et les travaux neufs. L'écart entre les deux courbes est plus important pour Marcoule qui a des installations plus anciennes et plus diversifiées, notamment des réacteurs. Ceci apparaît clairement dans la figure 11 qui montre la ventilation, en 1984, des doses collectives par nature d'activité.

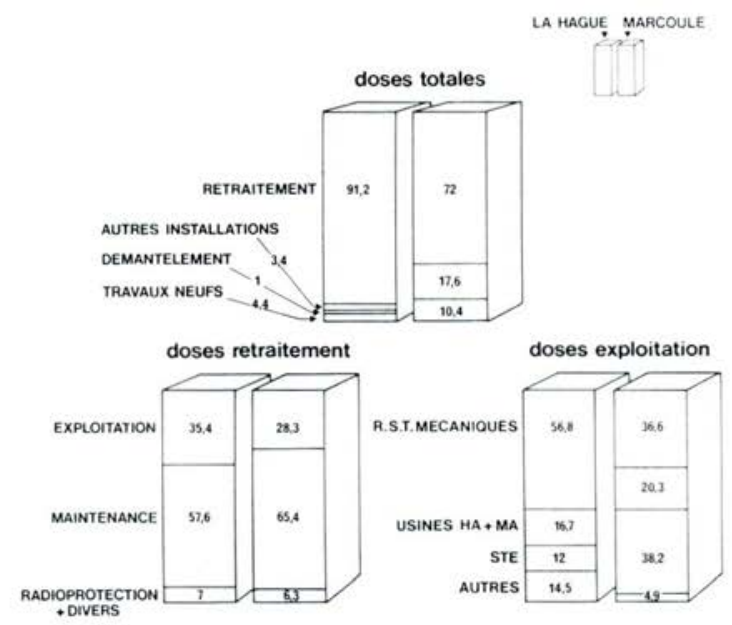

Fig. 11. - Ventilation des doses collectives 1984 en \% par nature d'activité.

On peut noter, en outre, pour les deux usines: la prépondérance des doses dues à la maintenance, le poids en exploitation des opérations mécaniques de tête, la contribution importante de la STE à Marcoule.

La figure 12 montre l'évolution très favorable des doses collectives totales (Cogéma + entreprises) rapportées à la production et exprimées en H.mSv par MWe.an-1 (échelle de gauche) ou GW.heure (échelle de droite). Le graphique ne concerne que l'usine de La Hague, qui est la seule à ne retraiter que des combustibles électrogènes. Les valeurs observées pour ces dernières années (environ $1 \mathrm{H} . \mathrm{mSv}^{\mathrm{M}} \mathrm{MWe} . \mathrm{an}^{-1}$ ) montrent que la contribution du retraitement, dans la dose collective totale des travailleurs du cycle de combustible, est relativement faible.

La figure 13 montre comment se répartissent la dose collective et les effectifs surveillés entre la Cogéma et les entreprises. L'évolution des doses moyennes annuelles est indiquée sur la figure 14 pour l'ensemble du personnel surveillé et sur la figure 15 pour la Cogéma d'une part et les entreprises d'autre part. Ces courbes montrent : 


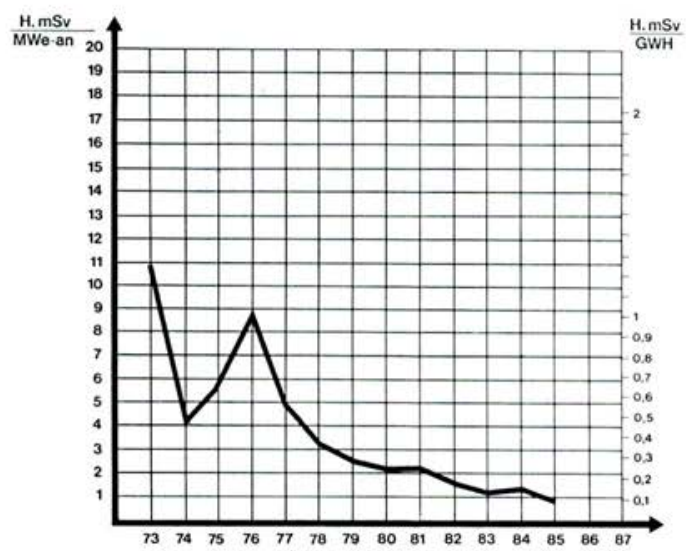

Fig. 12. - Doses collectives spécifiques (Cogéma + entreprises), La Hague, en H.mSv/MWe.an ${ }^{-1}$ et H.mSv/GWh.

- une décroissance satisfaisante des doses à partir des maximums observés en 1975 à La Hague (5 mSv) et en 1980 à Marcoule (3,5 mSv) qui se

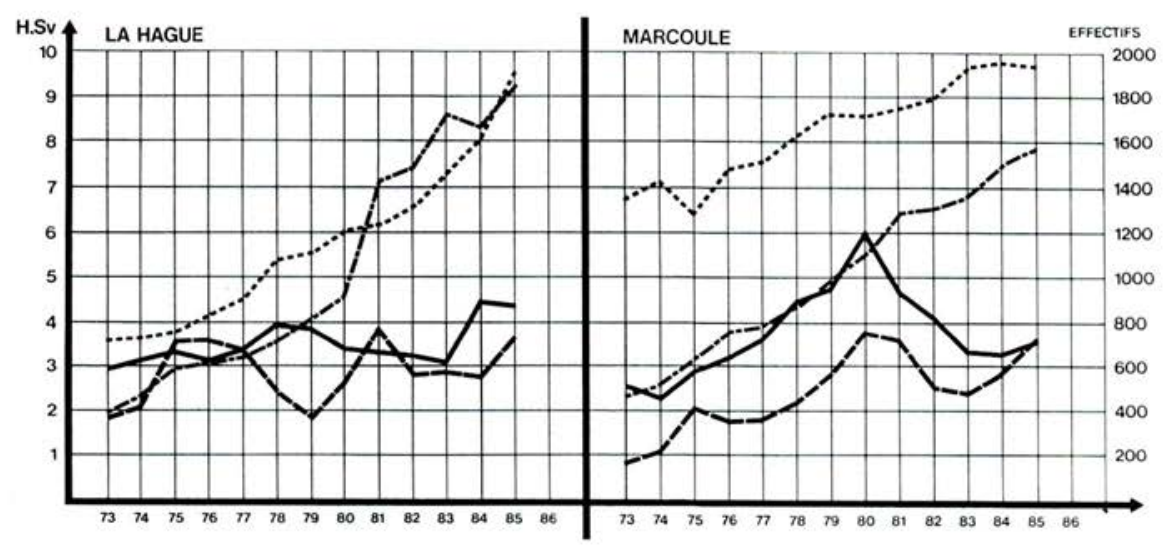

Fig. 13. - Répartition de la dose collective et des effectifs entre Cogéma et entreprises. 


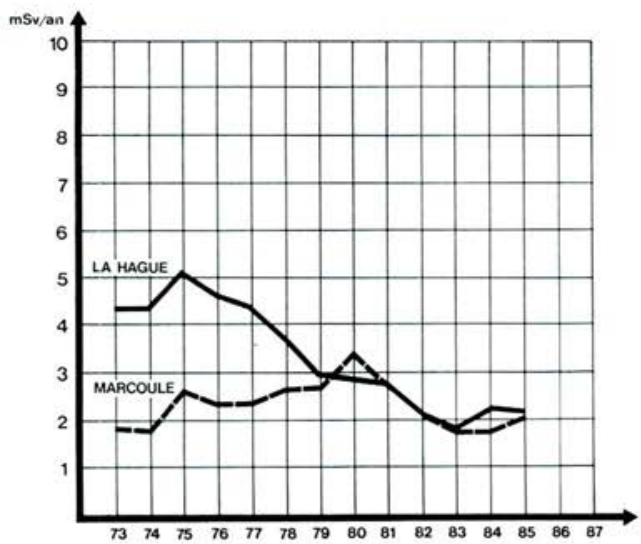

Fig. 14. - Doses moyennes annuelles en mSv (Cogéma + entreprises).

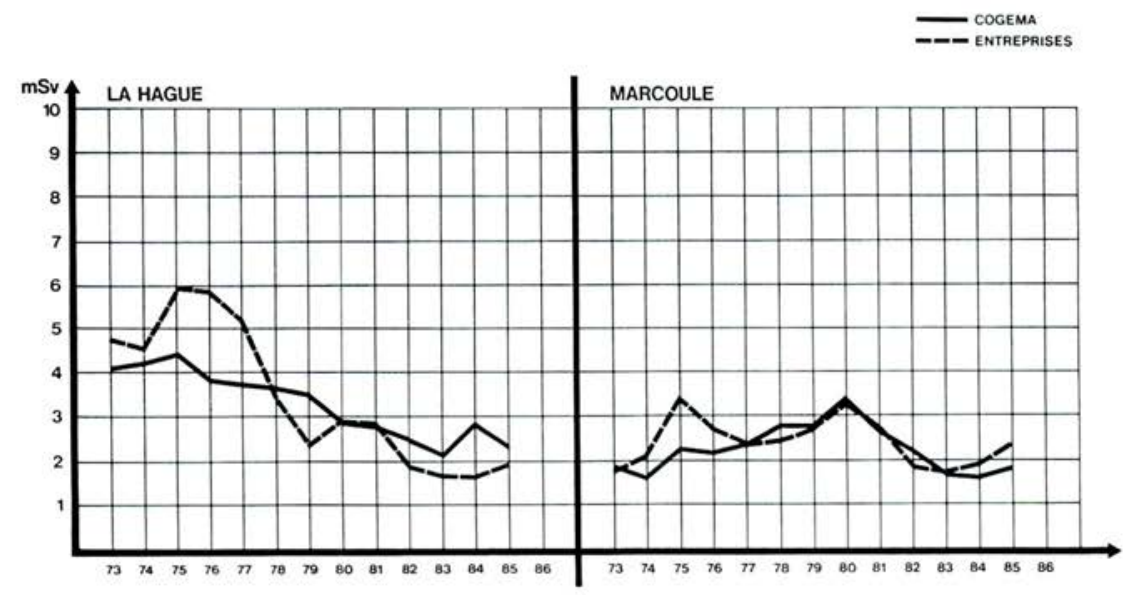

Fig. 15. - Doses moyennes annuelles en mSv, Cogéma et entreprises.

stabilisent depuis quelques années autour de $2 \mathrm{mSv}$ dans les deux établissements, soit $4 \%$ de la limite admissible pour les agents directement affectés aux travaux sous rayonnements ;

- une relative homogénéité entre les valeurs observées pour le personnel Cogéma et le personnel des entreprises extérieures.

La dernière figure 16 montre la valeur relative, exprimée en pourcentage des effectifs, du nombre d'agents se situant dans trois tranches de doses supérieures à $15 \mathrm{mSv} / \mathrm{an}: 15$ à 20, 20 à 30 et $>$ à $30 \mathrm{mSv} / \mathrm{an}$. Sans entrer dans le détail, on peut faire les remarques principales suivantes: 


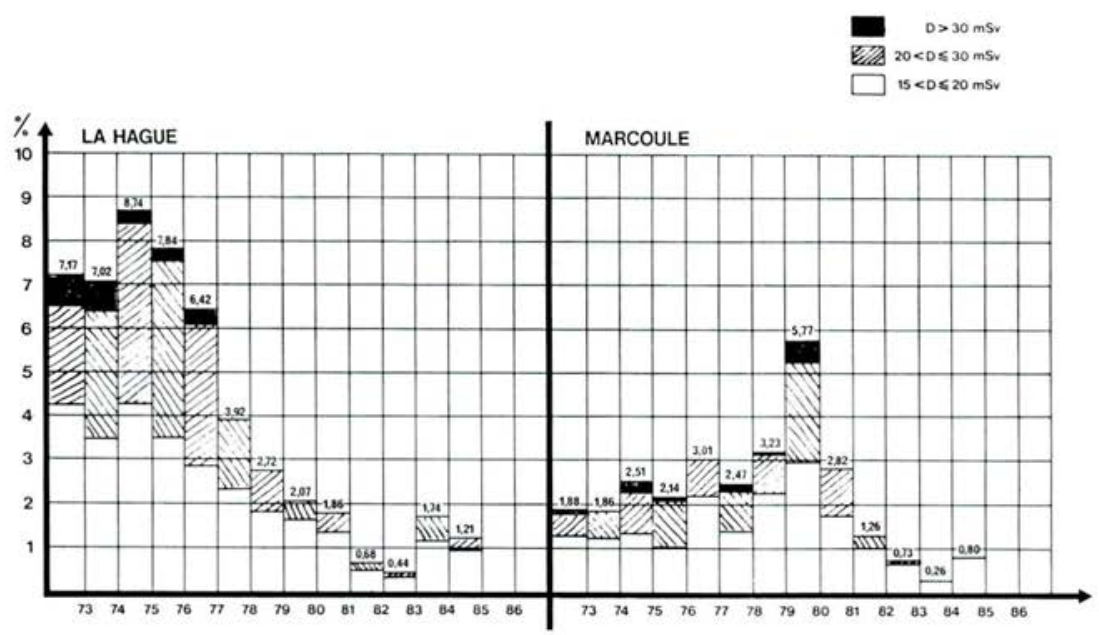

Fig. 16. - Répartition des effectifs en \% par tranches de doses supérieures à $15 \mathrm{mSv} \cdot \mathrm{an}^{-1}$.

- le nombre des agents ayant reçu une dose supérieure à 15 mSv dans l'année a atteint, comme les doses moyennes, sa valeur maximale en 1975 à La Hague $(8,7 \%$ des effectifs) et en 1980 à Marcoule $(5,8 \%)$. Actuellement, ces valeurs gravitent autour de $1 \%$, dont la majeure partie se situe dans la tranche 15 à $20 \mathrm{mSv}$;

- aucun agent, depuis 1978 à La Hague et depuis 1981 à Marcoule, n'a reçu une dose supérieure à $30 \mathrm{mSv}$. Lors des années antérieures, les quelques agents situés dans cette tranche de dose sont restés en dessous de la limite de 50 mSv.an-1.

Deux exceptions, concernant des irradiations accidentelles survenues sur le site de La Hague sont, toutefois, à signaler:

- en 1981, un agent d'entreprise, peintre en bâtiment, a reçu une dose de $57 \mathrm{mSv}$ alors qu'il travaillait à proximité d'un silo de déchets où un incident de combustion spontanée venait de se produire ;

- en 1982, un autre agent d'entreprise a reçu une dose de $212 \mathrm{mSv}$ au cours d'une opération de gammagraphie n'ayant rien à voir avec les activités de retraitement.

\section{CONCLUSION}

L'ensemble de ces résultats montrent que les difficultés rencontrées dans le retraitement ont été progressivement surmontées au cours des années et que cette activité se déroule maintenant dans des conditions de sécurité radiologique satisfaisantes. Cependant, pour les futures usines en cours de construction, la Cogéma s'est fixée des objectifs plus contraignants : 
Les deux usines UP2 800 et UP3, dont la capacité nominale sera quatre fois supérieure à celle de l'usine UP2 400 actuelle, sont, en effet, conçues de façon à ce qu'aucun agent ne reçoive en exploitation normale une dose supérieure à $5 \mathrm{mSv} / \mathrm{an}^{-1}$.

Cet objectif ambitieux peut être atteint grâce aux progrès technologiques et à l'expérience acquise qui a permis de dégager des principes directeurs. Les plus importants concernent les opérations de maintenance et les interventions qui, nous l'avons vu, contribuent à plus de $50 \%$ de la dose collective. En conséquence, les nouvelles installations sont conçues de façon à ce que :

- les opérations de maintenance et d'entretien puissent se dérouler comme des opérations d'exploitation normale :

- les opérations d'intervention puissent s'effectuer dans un minimum de temps et avec une exposition aussi faible que possible des intervenants.

Cela conduit à prévoir, dès la conception : la possibilité de vidange et de rinçage des capacités, l'accessibilité des cellules et des appareillages pour les hommes et les moyens mécaniques qui doivent être adaptés, les emballages nécessaires aux déchets et aux matériels à récupérer ainsi que leur cheminement dans l'installation, le confinement des zones de transfert, l'implantation d'un réseau de ventilation spécial, dit d'intervention.

Enfin, l'expérience a permis de dégager la nécessité d'une formation poussée du personnel sur le plan technique et sur les méthodes de travail en milieu ionisant, et d'une sensibilisation permanente de tous aux problèmes de sécurité par des recyclages et des exercices périodiques. 